\title{
Prognostic significance of HER2 expression in neuroblastic tumors
}

Ewa Izycka-Swieszewska ${ }^{1}$, Agnieszka Wozniak ${ }^{2,3}$, Jacek Kot ${ }^{4}$, Wieslawa Grajkowska ${ }^{5}$, Anna Balcerska ${ }^{6}$, Danuta Perek ${ }^{7}$, Bozena Dembowska-Baginska ${ }^{7}$, Teresa Klepacka ${ }^{8}$ and Elzbieta Drozynska ${ }^{6}$

${ }^{1}$ Department of Pathomorphology, Medical University of Gdansk, Gdansk, Poland; ${ }^{2}$ Department of Biology and Genetics, Medical University of Gdansk, Gdansk, Poland; ${ }^{3}$ Department of General Medical Oncology, Laboratory of Experimental Oncology, University Hospitals Catholic University Leuven, Leuven, Belgium;

${ }^{4}$ Department of Hyperbaric Medicine and Sea Rescue, Medical University of Gdansk, Gdynia, Poland;

${ }^{5}$ Department of Pathology Children's Health Memorial Institute, Warsaw, Poland; ${ }^{6}$ Department of Pediatrics, Hematology, Oncology and Endocrinology, Medical University of Gdansk, Gdansk, Poland; ${ }^{7}$ Department of Oncology, Children's Health Memorial Institute, Warsaw, Poland and ${ }^{8}$ Department of Pathology,

Institute of Mother and Child, Warsaw, Poland

HER2 is essential for normal embryonic development and has a critical function in oncogenesis and progression of some types of cancer. Neuroblastic tumors create a heterogenous group of pediatric embryonal tumors of sympathoadrenal lineage. The biological and prognostic function of HER2 in these tumors is not well established. In this study, we evaluated the status of HER2, its prognostic significance, and clinicopathological correlations in series of 79 untreated neuroblastoma. The immunohistochemical assessment of HER2 and Ki-67 (proliferation index) as well as HER2 copy number status were performed on tissue microarrays. HER2 expression characterized 63 tumors, including 34 with low and 29 with high level, showing either membranous or mixed membranous-cytoplasmic pattern. Sixteen cases were HER2 immunonegative. The pattern of immunolabeling depended on the maturity of neuroblastic cells, being the most intense in differentiating neuroblasts. None of the tumors revealed HER2 amplification. In the examined group, $20 \%$ of patients died of disease from 4 to 107 months (median 18) from the diagnosis, and the survivors were followed up for 14-149 months (median 59). Patients' age, stage of disease, tumor location, mitosis/karyorrhexis index (MKI), and presence of HER2 expression were statistically significantly related to survival probability as detected by the Cox proportional hazard model. In the univariate analysis, Kaplan-Meier curves revealed significantly poorer outcome of HER2 negative than HER2-positive tumors (either low or high expression). The immunonegativity was associated with adverse clinicopathological parameters, including poor survival, metastatic stage of disease, un- or poorly differentiated histology, high MKI, and higher proliferation index. In conclusion, HER2 expression, not accompanied by gene amplification, is common in neuroblastic tumors. HER2 positivity seems to have a positive prognostic significance. HER2 expression with a variable pattern is a marker of the stage of neuroblastic cells differentiation.

Modern Pathology (2010) 23, 1261-1268; doi:10.1038/modpathol.2010.115; published online 25 June 2010

Keywords: FISH; HER2 protein expression; HER2 status; neuroblastoma; patients' survival; prognostic factor; proliferation index

Correspondence: E Izycka-Swieszewska, MD, PhD, Department of Pathomorphology, Medical University of Gdansk, Debinki 7 Street, 80-211 Gdansk, Poland.

E-mail: eczis@wp.pl

Received 28 January 2010; revised and accepted 6 May 2010; published online 25 June 2010
Neuroblastic tumors are the most frequent extracranial infantile solid tumors and common pediatric neoplasms of the sympathetic ganglia and adrenal medulla. ${ }^{1-3}$ They develop from the persistent population of embryonal neural crest cells that would have either differentiated or undergo apoptosis. ${ }^{1-3}$ Neuroblastic tumors are remarkable for their diverse 
pathological picture concerning the level of neoplastic cells differentiation and Schwannian stroma amount as well as diverse ways of biological behavior ranging from spontaneous regression to the lethal metastatic spread. ${ }^{1-3}$ The new International Neuroblastoma Risk Group classification system uses relevant factors, including tumor stage, patients' age, histologic category, grade of tumor differentiation, the status of MYCN gene and chromosome $11 \mathrm{q}$ status as well as DNA ploidy. ${ }^{4}$ Nowadays, the status of these factors is necessary for the adjustment of therapeutic protocols ranging from the 'wait and see' strategy, throughout multimodal surgery, adjuvant chemotherapy, and radiotherapy up to megachemotherapy with bone marrow transplantation. ${ }^{3-5}$ About half of all cases are classified as high-risk with long-term survival rates $<40 \%$ despite intensive complex treatment. ${ }^{3,5}$ Better understanding of signaling pathways involved in neuroblastoma tumorigenesis and disease progression may allow to develop more effective therapies based on individualized tumor predicitive biomarkers profile.

More than 20 years ago, human epidermal growth factor receptor 2 (ERBB2, HER2) encoded by the HER2 gene was first identified as a transforming gene in ethylnitrosourea-induced rat neuroblastoma. ${ }^{6}$ This transmembrane glycoprotein-member of EGFRs tyrosine kinase receptors family mediates many cellular signaling pathways in developmental, physiological, and neoplastic processes. ${ }^{7-10}$ So far, there has been no HER2 ligand identified and the receptor acts on the way of homo- or heterodimerization with the other EGFR family receptors. ${ }^{7,11}$ HER2 has a pivotal function in embryogenesis dictating the establishment of several cell lineages through mesenchyme-epithelial-neuroectodermal inductive processes. ${ }^{8,9,12}$ It influences cellular migration, differentiation, and interactions between cells. $^{8-10,12}$ The critical function of ERBB2 is exemplified in murine erbb2 mutants, which display an abnormal structure of heart, peripheral nervous system, and sympathetic ganglia, absence of Schwann cells, and lack of functional neuromuscular junction. ${ }^{8,12}$

The tumorigenic potential of abnormally activated HER2 is supported by many experimental models. $^{7,11,13}$ HER2 upregulates cellular proliferation and migration by sustained activation of multiple signaling pathways, including mitogen-activated protein kinase and phosphoinositide-3-kinas/AKT cascades. $^{7,9,11}$ Amplification of HER2 gene and overexpression of the encoded protein are associated with a poor prognosis in several types of cancer. ${ }^{7,11,14}$ Based on that, selective HER2 inhibitors have been successfully introduced in breast cancer and have been tested in other tumor types as the target therapy. ${ }^{7,11,14,15}$ On the other hand, in many tumors, HER2 expression does not influence the patients' survival or even less frequently is a favorable prognostic factor. ${ }^{13,16,17}$
The results for HER2 expression and its biological function in neuroblastic tumors are sparse and controversial. The aim of this study was to evaluate the status of HER2 in our series of neuroblastoma and to assess its prognostic significance and clinicopathological correlations.

\section{Materials and methods}

A group of 79 patients with neuroblastic tumors diagnosed and treated between 1996 and 2007 in three pediatric oncological centers in Poland (Department of Pediatrics, Hematology, Oncology and Endocrinology, Medical University of Gdansk; Department of Pediatric Oncology Children's Health Memorial Institute; and Institute of Mother and Child in Warsaw) was included in the study, based on availability of tissue material. Clinical data comprised patients' age, primary tumor localization, stage of disease, and overall survival at the time of the last observation (March 2009). Molecular data included tumor cell ploidy and MYCN status.

\section{Immunohistochemistry}

The study was performed on the archival material of routinely processed tumor sections taken at the surgical biopsy or tumor resection before chemotherapy. The hematoxylin and eosin-stained slides were reviewed by two independent pathologists (EIS and WG) to verify the diagnosis, establish mitosis/ karyorrhexis index (MKI), and choose the representative sections for further evaluation. In the next step, the tissue microarrays (TMAs) were constructed using Tissue-Tek Quick-Ray Tissue Microarray System (Sakura Finetek USA) according to the manufacturer's instructions. One or two $5 \mathrm{~mm}$ core biopsies were obtained for each tumor and mounted into recipient blocks.

The following antibodies were used for immunohistochemical evaluation: C-terminal c-erbB-2 (pc. rabbit, 1:50, DAKO, A 0485), external domain of c-erbB-2 (mc. Novocastra 10A7), and Ki-67 (1:100, DAKO, F 0788), with appropriate positive and negative controls. A standard protocol with heatinduced antigen retrieval in citrate buffer was performed. Subsequently, the slides were incubated with the primary antibody for $30 \mathrm{~min}$ in a room temperature. Thereafter, visualization system En Vision and DAB (DAKO) with hematoxylin staining were performed.

HER2 expression was analyzed with both c-erbB-2 antibodies in neuroblastic cell component of tumors in a descriptive and semiquantitative method. Some cases contained surrounding suprarenal gland that was used for the internal control. We also examined five normal paraspinal ganglia (taken at the autopsy) as well as two spontaneously aborted fetuses $(10,13$ th week of the pregnancy). In the quantitative analysis, the degree of membranous or 
membranous-cytoplasmic HER2 labeling was assessed. Expression level was estimated with our own score system based on the percentage of positive cells and staining intensity. The labeling in $<5 \%$ of neoplastic cells was considered negative (score 0 ), immunopositivity of $5-50 \%$ of cells was scored as 1 , and score 2 concerned cases with $>50 \%$ positive cells. The labeling intensity was graded as follows: negative (0), low (1), intermediate (2), and strong (3). The final expression level was counted as the sum of the above-mentioned parameters: 0 and 1 were considered HER2 negative, 2 and 3 reflected low expression, whereas 4 and 5 were considered a high HER2 expression.

Ki-67 expression was assessed to establish the proliferation index (PI). The quantitative study was carried out semiautomatically using the image analyzer system (microscope BX 51 and digital camera SP-350 with Quick Photo Camera 2.2. software, Olympus). Tumor fields with the highest number of the Ki-67 positive nuclei were identified and total number of nuclei and immunopositive nuclei was manually pointed and counted on the computer images at $\times 200$ magnification. The proportion of positive tumor cells was measured for at least 100-1000 cells depending on tumor histology and PI was reported in the percentage of positive cells.

A fluorescence in situ hybridization (FISH) was performed to estimate HER2 copy number. The hybridization with SpectrumOrange LSI EGFR/ SpectrumGreen CEP 17 probe set (Abbott Molecular) was carried out following the manufacturer's protocol. For FISH analysis, 40-100 non-overlapping nuclei were scored for signals from both DNA probes using a fluorescent microscope with SpectrumOrange/SpectrumGreen/DAPI set of filters (Axioskop, Zeiss) at a magnification of $\times 100$. The images were recorded by conjugated CCD camera and were further analyzed with the software CytoVysion Ultra System (Applied Imaging International). The presence of HER2 gene clusters in $>10 \%$ of analyzed cells or ratio of HER2 to CEP17 of $>2.2$ was considered gene amplification.

Table 1 Clinicopathological data of the analyzed group of neuroblastic tumors

$\begin{array}{ll}\text { Patients' age } & \text { 1-169 months (median 25) } \\ \text { Stage } & \text { I: 10, II: 13, III: 30, IV: 21, IV: 5 } \\ \text { Tumor localization } & \text { Suprarenal gland: 36, retroperitoneal } \\ & \text { space: 18, mediastinum: 16, sacral region: } \\ & \text { 3, paravertebral: 2, neck: 2, primary } \\ \text { disseminated: 2 } & \text { 56 Schwannian stroma poor } \\ \text { Tumor histology } & \text { (3 undifferentiated, 35 poorly differentiated, } \\ & \text { 18 differentiating) } \\ & \text { 15 Schwannian stroma rich } \\ & \text { (10 intermixed, 5 nodular } \\ & \text { ganglioneuroblastoma) } \\ & \text { 8 Schwannian stroma predominant } \\ & \text { (ganglioneuroma maturing) } \\ & \text { 25 low, 26 intermediate, 20 high } \\ & \end{array}$

\section{Statistical Analysis}

In the univariate analysis, the $\chi^{2}$ test and MannWhitney test were used to compare categorical and continuous variables, respectively. The relation between continuous and ordinal variables was analyzed with the Spearman's rank correlation. The interobserver agreement in immunohistochemistry assessment was measured with the $\kappa$ statistics. Survival analysis was performed with the KaplanMeier method, and survival between groups was compared using the log-rank test. In the multivariate analysis, the Cox proportional hazard model was used to identify prognostic factors in a total patients group. Data were expressed as median and range. The value of $P<0.05$ was interpreted as statistically significant. Analyses were performed with the software package STATISTICA (StatSoft, 2007, USA).

\section{Results}

The analyzed group of patients consisted of 79 children with neuroblastic tumors. MYCN amplification was reported in 10 tumors. DNA ploidy was known in 73 tumors, including 28 diploid, 41 triploid, 3 tetraploid, and 1 pentaploid case. The clinicopathological data are presented in Table 1.

Sixteen patients $(20.2 \%)$ died of disease in the period of 4-107 months (median 18) from the initial diagnosis (complete observations). The rest of the children lived for 14-149 months from initial diagnosis, with a median follow-up of 59 months to the observation at the database closure (censored observations). The Kaplan-Meier curve for the survival probability for the whole analyzed group is presented in Figure 1.

\section{Immunohistochemistry}

The labeling with both HER2 antibodies was similar, still monoclonal antibody showed slightly higher

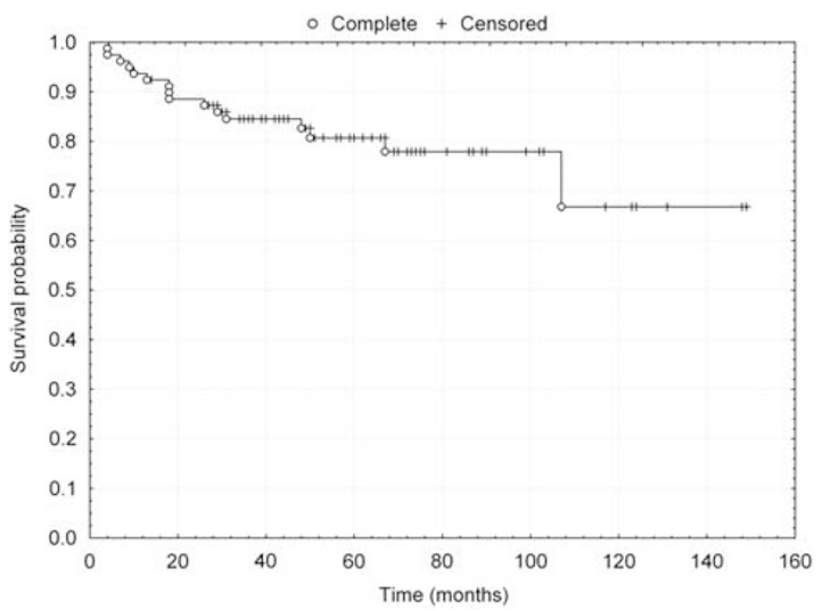

Figure 1 Kaplan-Meier curve for survival probability for the entire analyzed group $(n=79)$. 
membranous accentation than polyclonal one. The $\kappa$ statistics concerning the final expression level between both HER2 antibodies used was high $(\kappa=0.92)$. In normal suprarenal gland, HER2 was strongly positive in medulla and negative in adrenal cortex. The ganglion cells of normal autonomic ganglia showed low, mainly membranous and axonal expression and very low or even absent expression within the stroma. In fetal paravertebral ganglia, HER2 membranous/cytoplasmic expression characterized ganglioid cells and their axons.

In the examined group, 16 tumors were HER2 immunonegative, whereas 63 cases presented HER2 labeling with either membranous or mixed membranous-cytoplasmic pattern. Among HER2 positive, 34 tumors showed low and 29 high protein expression. Thirty-five cases were available for FISH analysis. No evidence of HER2 amplification was found in any tumor. The HER2/CEP17 was between 0.93 and 2.16 (mean 1.20). PI assessed with Ki-67 ( $\kappa=0.91)$ ranged from 0 to $72 \%$ (median $19 \%$ ).

The way of immunolabeling was associated with the maturity of neuroblastic cells (Figure 2). The undifferentiated and poorly differentiated neuroblasts were usually negative. HER2 expression was increasing in differentiating cells, with a maximal intensity in intermediate ganglioid cells. Mature neurons labeling was low membranous or cytoplasmatic and was present within the axons. Every tumor contained a population of immunonegative cells. The Schwannian stroma was weakly positive.

\section{Statistical Analysis}

In the univariate analysis, Kaplan-Meier curves revealed poorer outcome for diploid tumors $(P<0.004), M Y C N$-amplificated tumors $(P<0.001)$, metastatic stage $(P<0.001)$, age older than 18 months $(P<0.01)$, and high MKI $(P<0.001)$. HER2negative tumors had poorer outcome than either low or high HER2 expression (log-rank test, $P=0.005)$ as well as HER2-negative vs -positive cases $(P=0.008)$ (Figure 3$)$.

HER2 expression was significantly correlated with tumor stage divided into non-metastatic and metastatic, where HER2-negative cases were more often in the metastatic $(9 / 21,42.9 \%)$ than in a localized group $(7 / 58,12.1 \% ; P=0.03)$. Tumor histology was related to HER2 expression (positive vs negative), which differed between histopathological groups $(P<0.001)$, with a strong correlation between both parameters $(R s=0.49 ; \quad P<0.001)$ (Table 2). Moreover, HER2 status (positive vs negative) significantly differed between MKI groups $(P<0.001)$, with a strong correlation $(R s=-0.45$; $P<0.001)$. The differences were also significant concerning the incidence of HER2 and MKI $(P<0.001)$ (Table 2), with their correlation $(R s=-0.32 ; P=0.04)$. Next finding was different PI in tumors with different HER2 expression (negative vs positive, negative vs low expression, negative vs high expression: in all $P<0.001$ ) (Figure 4). PI did not differ between low and high HER2 expression group $(P>0.05)$; however, all these values were significantly correlated $(R s=-0.37 ; \quad P<0.001)$. Among 16 children who died, there were $7 / 16$ immunonegative, $6 / 34$ with low and $3 / 29$ with high HER2 expression.

Other relations were insignificant. MYCN nonamplified tumors were more often HER2 positive than amplified tumors (82.9 and $55.6 \%$, respectively), but this difference was of borderline significance $(P=0.055)$. No correlation between HER2 expression either with patients' age, tumor location, or tumor ploidy status was found.

From all analyzed parameters (ploidy, MYCN amplification, age, histology, HER2, stage, tumor location, MKI, and Ki-67) only age, stage, tumor location, MKI, and HER2 were statistically significantly related to survival probability in multivariate analysis as detected by the Cox proportional hazard model (Table 3).

\section{Discussion}

Neuroblastic tumors are heterogenous both clinically and biologically. ${ }^{1,4,18}$ Pathogenesis of neuroblastoma as the embryonal tumor is connected to the deregulation of normal developmental processes of sympathetic system. The neurotrophin pathways of TRK family tyrosine kinase receptors, which influence cellular survival, growth, and differentiation are one of the most explored there. ${ }^{18,19}$ Many additional signaling pathways affect biology of this tumor and their understanding might lead to the identification of novel therapeutic approaches. ${ }^{1,3}$ HER2, which is involved in developmental processes, tissue proliferation, and oncogenesis, represents one of the first validated therapeutic targets of an adult cancer. ${ }^{7,11,13}$ It interacts together with EGFR, HER3, and HER4 through a complex cellular signaling network with the hierarchical catalytic importance of HER2., ${ }^{7,8,13}$ Activated HER receptors cooperate with numerous downstream cytoplasmic effector proteins, depending on specific dimerization partners, which generate additional signal diversification. ${ }^{7,8,11}$ In embryogenesis, HER receptors display a temporal and topographical distribution with stage and tissue-dependent expression. ${ }^{6,10,13}$ During the development of autonomic nervous system, HER2 provides an essential coreceptor for HER3. ${ }^{9,10,12}$ It drives the migrating precursors to pause at a specific location and makes cells competent to receive differentiation signals and triggers the development of Schwann cells precursors. ${ }^{8,9,10,12}$ HER2 can be detected in neural and connective tissue during a relatively short embryonic period, whereas in some other tissues, it persists into adulthood. ${ }^{6,12}$ HER2 expression in different 

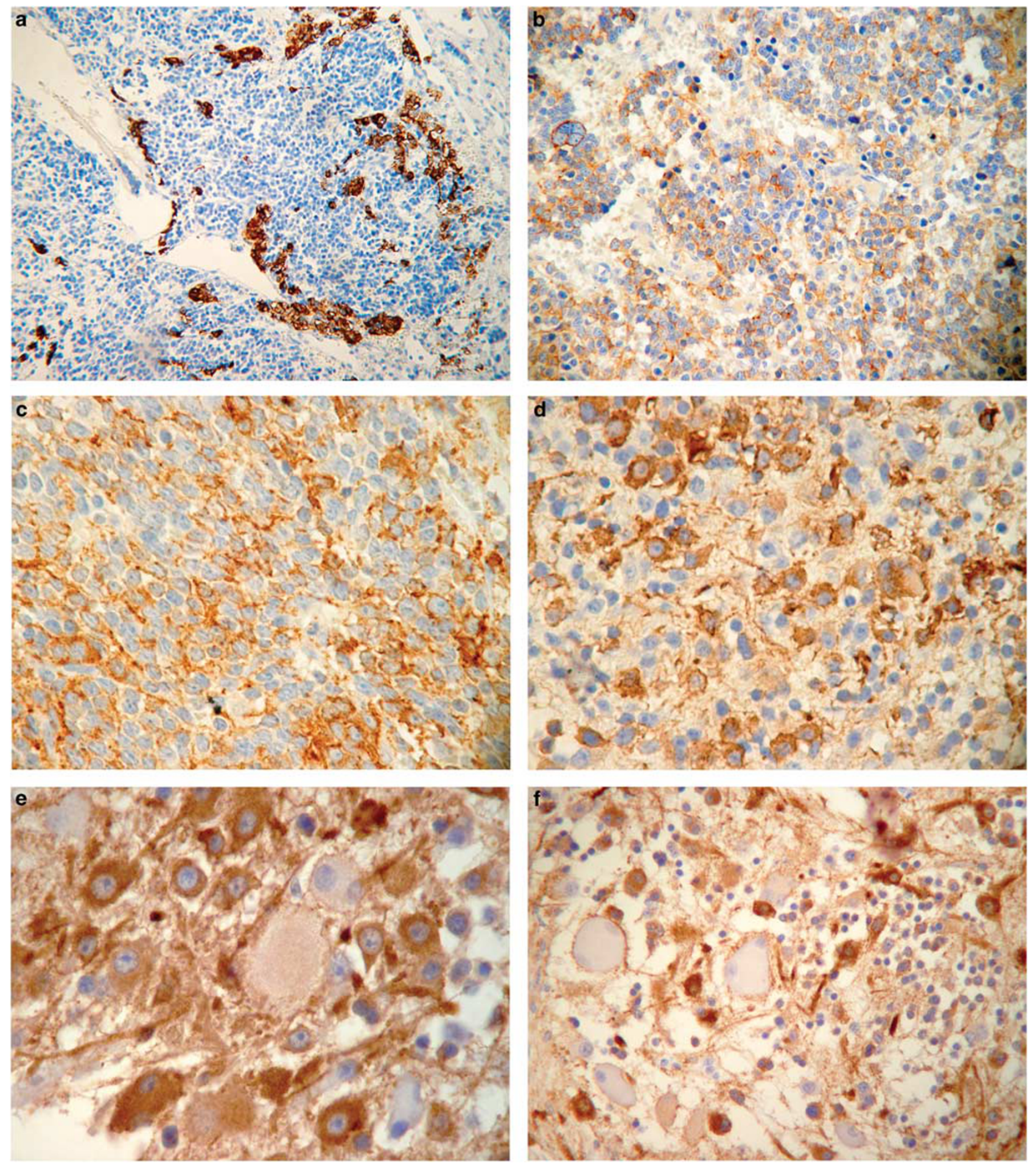

Figure 2 HER2 expression. (a) HER2-positive adrenomedullary cells and infiltration of immunonegative undifferentiated neuroblastoma $(\times 100)$. (b) Low membranous HER2 expression within poorly differentiated neuroblastoma $(\times 200)$. (c, d) Strong membranocytoplasmatic labeling in a population of differentiating neuroblasts $(\times 400)$. (e) Strong expression within ganglioid cells and low membranous expression within the ganglion cells $(\times 400)$. (f) Different pattern of HER2 expression within the neuroblastic cells with different stage of maturation $(\times 200)$.

normal and neoplastic tissues has been reported as membranous, cytoplasmatic, or combined. ${ }^{6,13,20,21}$ HER2 is regulated by many adaptor and scaffolding proteins; for instance erbin, which controls the receptor sorting. ${ }^{7,11,22}$ The example being membranous expression in keratinocytes and upper suprabasal layer of normal skin, but cytoplasmic in basal layer. ${ }^{22}$ In thyroid cancer, decreasing HER2 expression characterized dedifferentiated parts of poorly differentiated tumors up to immunonegativity in 


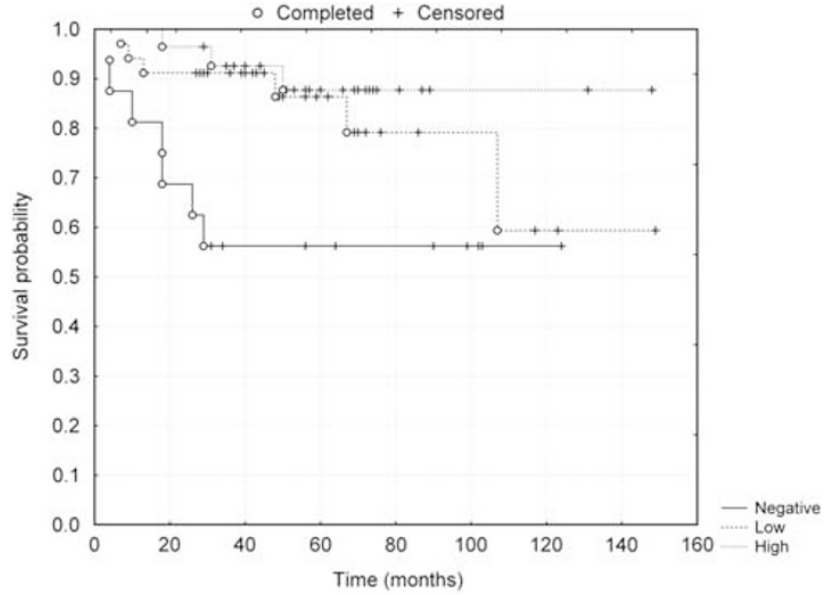

Figure 3 Survival probability between different levels of HER2 expression (log-rank test, $P=0.005)$. In the post hoc analysis, statistically significant differences between negative and both low and high expression $(P=0.014, P=0.005$, respectively), but not low vs high expression $(P=0.53)$ were present.

Table 2 HER2 expression (negative vs positive) for different histopathological groups

\begin{tabular}{lcc}
\hline HER2 negative (16) & $\begin{array}{c}\text { HER2 positive (63) } \\
\text { (low + high expression) }\end{array}$ \\
\hline $\begin{array}{c}\text { Histology } \\
\text { UN }\end{array}$ & $3(100.0 \%)$ & $0(0.0 \%)$ \\
PD & $12(34.3 \%)$ & $23(16+7)(65.7 \%)$ \\
DIF & $1(5.6 \%)$ & $17(6+11)(94.4 \%)$ \\
GNB & $0(0.0 \%)$ & $15(5+10)(100.0 \%)$ \\
GN & $0(0.0 \%)$ & $8(7+1)(100.0 \%)$ \\
MKI & & \\
Low & $3.0 \%$ & $97.0 \%$ \\
Inter & $19.2 \%$ & $80.8 \%$ \\
High & $50.0 \%$ & $50.0 \%$ \\
\hline
\end{tabular}

Un, undifferentiated; PD, poorly differentiated; DIF, differentiating; GNB, ganglioneuroblastoma; GN, ganglioneuroma.

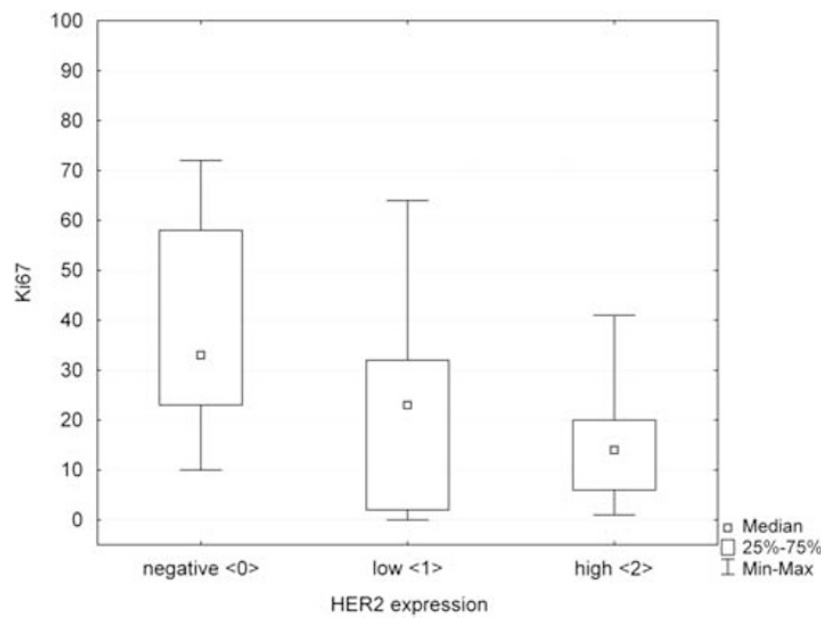

Figure 4 Proliferation index based on Ki-67 positivity between different HER2 expression groups (negative $<0>$ vs low $<1>$ vs high $<2>$ )
Table 3 Multivariate analysis of selected statistically significant parameters by the Cox proportional hazard model

\begin{tabular}{lcc}
\hline Parameter & OR $(-95 \%$ CI to 95\% CI) & $\mathrm{P}$ \\
\hline $\begin{array}{l}\text { Age (under 18, over 18 } \\
\text { months) }\end{array}$ & $28.809(4.145$ to 200.230$)$ & $<0.001$ \\
$\begin{array}{l}\text { HER2 (negative, } \\
\text { positive(low+high)) }\end{array}$ & $0.310(0.098$ to 0.978$)$ & $<0.05$ \\
$\begin{array}{l}\text { Stage (I, II, III, IV, IV) } \\
\begin{array}{l}\text { Tumor location } \\
\text { (extraadrenal, adrenal) }\end{array}\end{array}$ & $9.783(2.276$ to 42.054$)$ & $<0.003$ \\
MKI (low, intermediate, high) & $3.404(1.246$ to 9.297$)$ & $<0.02$
\end{tabular}

anaplastic carcinoma. ${ }^{17}$ In our study, we observed membranous-cytoplasmic HER2 labeling in normal adrenomedullary tissue, developing fetal autonomic neural crest cells and differentiating cells in neuroblastoma. Mature neurons of dorsal roots and sympathetic ganglia revealed membranous rimming.

Numerous experimental models prove the hypothesis of HER2-mediated tumorigenesis in some types of cancer, especially in cooperation with HER3. .,11,13,14 HER2 expression has been studied most extensively in breast cancer, where the protein overexpression is detected in about $25 \%$ of cases. Moreover, ERBB2 strong membranous labeling correlates there with gene amplification, poor prognosis, and higher PI. ${ }^{7,14,23}$ HER2 overexpression concerns also about $20 \%$ of gastric cancer and several percent of prostate and ovarian cancer. ${ }^{7,11,24}$ This receptor has become the target for anticancer therapies in so-called 'HER2-driven' cases. Several approaches, including monoclonal antibodies blocking the extracellular domain or small molecule intracellular kinase inhibitors, are currently being used in clinical practice. . $^{71,15}$

It is worth pointing out that in most tumors, the incidence of HER2 expression and its clinical relevance is controversial and not well established. ${ }^{11,13}$ The data on HER2 expression in childhood cancer is sparse and there is a lack of general consensus regarding its oncogenic function, possible prognostic impact, and therapeutic utility. ${ }^{13}$ However, the first clinical trials on EGFR and HER2 target therapy in short series of miscellaneous refractory pediatric tumors have been introduced. ${ }^{13,25}$ Osteosarcoma was described in a wide range from a few to $60 \%$ of immunopositive cases without the influence of protein overexpression on target therapeutic effectiveness. ${ }^{21}$ The study of Salem et $a l^{16}$ on Wilms tumors showed HER2 immunonegativity in high-risk cases with blastemal predominance. The uneven distribution of HER2 in histologic components of nephroblastoma suggested its contribution to the differentiation and generation of epithelial component. ${ }^{16}$ In medulloblastomas, HER2 membrano-cytoplasmic expression is frequent and in coexpression with HER4 serves as an adverse prognostic factor. ${ }^{13}$ Rhabdomyosarcomas in $22 \%$ of cases showed HER2 membranous labeling with an frequent cytoplasmic pattern, without gene 
amplification or correlation with age of patients and stage of disease. ${ }^{26}$

HER2 expression in human neuroblastoma was the subject of a few studies. Some discrepancies of the results are probably caused by diverse immunohistochemical assessment, different series of tumor subtypes, and variable antibody clones. In 1995, Layfield et $a l^{27}$ found HER2 expression as a poor prognostic factor in a group of 31 tumors. The authors distinguished only strong HER2 positivity and immunonegativity. Median survival in four immunopositive cases was 12 months comparing to 138 months in 27 negative cases. No associations with other patho-clinical parameters (stage, histology, DNA ploidy, PI) were detected..$^{27}$ The same year, Goji et $a l^{20}$ described HER2 expression in $\sim 60 \%$ of 62 neuroblastic tumors with two staining patternsfocal cytoplasmic and diffuse membranous. Western blot analysis proved cytoplasmic staining due to full-length protein. No correlation with histology and clinical stage was revealed, but the number of patients with MYCN amplification was higher in the immunonegative group. Performed immunoelectron microscopic examination of developing sympathetic ganglions localized cytoplasmic HER2 labeling on polysomes associated with endoplasmic reticulum. ${ }^{20}$ In 2003, Gambini et $a l^{28}$ examined 93 neuroblastic tumors and 16 cell lines with Hercept test. They respected only membranous labeling, showing $14 \%$ strongly positive, $7 \%$ low expression, and the rest of negative cases. No relations with patho-clinical data (age, stage, sex, ploidy, histology, overall, and event-free survival) were revealed. ${ }^{28}$ Next, Ho et $a l^{29}$ studied the expression and function of the EGFR family proteins and their pathways in cell lines and 18 neuroblastic tumors by semiquantitative RT-PCR and immunoblotting. They found readily detectable levels of HER2 in majority of cases without the correlation with tumor stage. ${ }^{29}$

In our survey of 79 tumors, we analyzed both membranous and cytoplasmic labeling, according to the phenomenon that in pediatric tumors, HER2 expression is frequently cytoplasmic. ${ }^{13,16,26}$ Sixteen cases were HER2 immunonegative, 34 showed low and 29 high expression. Performed FISH analysis disclosed no evidence of HER2 amplification in the studied group. It is known that although many pediatric solid tumors contain detectable HER2 RNA and protein levels, they very rarely show gene amplification. $^{13,21}$ This suggests the alternative way for HER2 activation and a biological function in childhood cancer. ${ }^{13}$

In the presented group, Cox proportional hazard model showed statistically significant relation between survival probability and some widely accepted prognostic factors, including age, stage, tumor location, and MKI. We found for the first time HER2 as an independent prognostic factor. Lack of HER2 expression strongly associated with higher mortality and showed a poor outcome of these tumors, both in uni- and multivariate analysis.
Moreover, we revealed statistically significant correlations between HER2 expression and some clinicopathological parameters. HER2 negativity was associated with some poor prognostic factors, including metastatic stage, high MKI, low differentiation, and higher PI. HER2 expression was also significantly related to tumor histology, being negative or low in undifferentiated and poorly differentiated tumors. High expression characterized mainly differentiating neuroblastomas and ganglioneuroblastomas. The pattern of immunolabeling also varied among the cells within the tumor. It was negative in the most primitive cells, mixed membrano cytoplasmic in most neuroblastic cells with features of differentiation and membranous in some poorly differentiated cells and mature ganglion cells. We share the opinion of Goji et $a l^{20}$ and Gambini et $a l^{28}$ who suggested that the way of HER2 expression reflects the phenotype of developing neurons, which may follow a variety of stages of neural crest cell lineages. ${ }^{19}$ Most probably, one of the biological functions of HER2 in neuroblastic tumors is connected to differentiation. On the other hand, the low membranous expression in maturing and mature ganglion cells might be necessary for their survival or their specific functions through various signaling pathways.

An important component of neuroblastic tumors is Schwannian stroma, which interacts with neuroblastic cell component. ${ }^{1,2,30}$ All immunonegative tumors in our study came from the Schwannian stroma poor group. Although HER2 is necessary for Schwann cell genesis in normal embryonal development,, ${ }^{8,9,12}$ it seems that neuroblastic cells lacking HER2 expression are unable to trigger the Schwann cells development within the tumor. Possibly, it has further consequences due to the lack or reduced specific cross-talk between both neuroblastoma components.

In our study, HER2 expression was significantly correlated with MKI and PI (Ki-67). Most often, immunonegative tumors had high MKI, whereas HER2-positive tumors had usually low or intermediate MKI. PI in examined group ranged between 0 and $72 \%$ (median 19\%), with significantly higher values in HER2-negative tumors (median 32\%) than in the immunopositive group (median 13\%). There was no difference in PI between low and high HER2 expression groups. In earlier studies on neuroblastoma, higher PI was related to poor prognosis and lower differentiation, ${ }^{31}$ but the significant relations with HER2 have not been described before. ${ }^{27-29}$ Our next finding was that HER2-negative cases were significantly more frequent in metastatic disease. We also observed more frequent $M Y C N$ amplification in negative cases, similarly to the results by Goji et al. ${ }^{20}$ No other associations of HER2 expression with established prognostic factors were found.

In conclusion, HER2 expression in neuroblastic tumors is common, but it is not due to the gene amplification. HER2 expression in neuroblastoma seems to have a positive prognostic significance. 
The immunonegativity is associated with adverse patho-clinical parameters, including poor survival, metastatic stage of disease, un- or poorly differentiated histology, high MKI, and higher PI. HER2 expression with a variable pattern is a marker of the stage of ganglionic-neuronal differentiation and seems to be related to Schwannian stroma development. All the above suggests HER2 function in complex tumorigenesis; however, the ways and usefulness of therapeutic targeting of this molecule in neuroblastoma needs further investigation.

\section{Acknowledgements}

This study was supported by grant from Polish Ministry of Science and Higher Education (N401 176 31/3867). We thank Anita Matyskiel for her excellent technical support and Dr Grazyna KobierskaGulida for helpful discussion.

\section{Disclosure/conflict of interest}

The authors declare no conflict of interest.

\section{References}

1 Brodeur G. Neuroblastoma: biological insights into a clinical enigma. Nat Rev Cancer 2003;3:203-216.

2 Shimada H, Ambros I, Dehner L, et al. The International Neuroblastoma Pathology Classification (the Shimada System). Cancer 1999;86:364-372.

3 Maris J, Hogarty M, Bagatell R, et al. Neuroblastoma. Lancet 2007;369:2106-2120.

4 Cohn S, Pearson A, London W, et al. The International Neuroblastoma Risk Group (INRG) classification system: an INRG Task Force report. J Clin Oncol 2009;27:289-297.

5 Fong A, Park J. High-risk neuroblastoma: a therapy in evolution. Pediatr Hematol Oncol 2009;26:539-548.

6 Kokai Y, Cohen J, Drebin J, et al. Stage and tissuespecific expression of the neu oncogene in rat development. Proc Natl Acad Sci 1987;84:8498-8501.

7 Moasser M. The oncogene HER2: its signaling and transforming functions and its role in human cancer pathogenesis. Oncogene 2007;26:6469-6487.

8 Chan R, Hardy W, Laing M, et al. The catalytic activity of the ErbB-2 receptor tyrosine kinase is essential for embryonic development. Mol Cell Biol 2002;22:1073-1078.

9 Casalini P, Iorio M, Galmozzi E, et al. Role of HER receptors family in development and differentiation. J Cell Physiol 2004;200:343-350.

10 Shilo B. Regulating the dynamics of EGF receptor signaling in space and time. Development 2005;132: 4017-4027.

11 Baselga J, Swain S. Novel anticancer targets: revisiting ERBB2 and discovering ERBB3. Nat Rev Cancer 2009; 9:463-475.

12 Britsch S. The neuregulin-I/ERBB signaling system in development and disease. Adv Anat Embryol Cell Biol 2007;190:1-65.
13 Gilbertson RJ. ErbB2 in pediatric cancer: innocent until proven guilty. Oncologist 2005;10:508-517.

14 Ross J, Fletcher J, Linette G, et al. The Her-2/neu gene and protein in breast cancer: biomarker and target of therapy. Oncologist 2003;8:307-325.

15 Jones KL, Buzdar AL. Evolving novel anti HER2 strategies. Lancet Oncol 2009;10:1179-1187.

16 Salem M, Kinoshita Y, Tajiri T, et al. Association between the HER2 expression and histological differentiation in Wilms tumor. Pediatr Surg Int 2006; 22:891-896.

17 Ensinger C, Prommegger R, Kendler D, et al. HER2/ neu expression in poorly-differentiated and anaplastic thyroid carcinomas. Anticancer Res 2003;23: 2349-2353.

18 Brodeur G, Minturn J, Ho R, et al. Trk receptor expression and inhibition in neuroblastoma. Clin Cancer Res 2009;15:3244-3250.

19 Huber K. The sympathoadrenal cell lineage: specification, diversification, and new perspectives. Dev Biol 2006;298:335-343.

20 Goji J, Nakamura H, Ito H, et al. Expression of c-ERbB2 in human neuroblastoma tissues, adrenal medulla adjacent to tumor, and developing mouse neural crest cells. Am J Pathol 1995;146:660-672.

21 Somers G, Ho M, Zielenska M, et al. HER2 amplification and overexpression is not present in pediatric osteosarcoma: a tissue microarray study. Ped Dev Pathol 2005;8:525-532.

22 Lebeau S, Masouye I, Berti M, et al. Comparative analysis of the expression of ERBIN and Erb-B2 in normal human skin and cutaneous carcinomas. Br J Dermatol 2005;152:1248-1255.

23 Ruiz C, Seibt S, Al Kurava K, et al. Tissue microarrays for comparing molecular features with proliferation activity in breast cancer. Int J Cancer 2006;118: 2190-2194.

24 Tuefferd M, Couturier J, Penault-Llorca F, et al. HER2 status in ovarian carcinomas: a multicenter GINECO study of 320 patients. PloS ONE 2007;11:e1138.

25 Jakacki R, Hamilton M, Gilbertson R, et al. Pediatric phase I, and pharmacokinetic study of Erlotinib followed by the combination of Erlotinib abd Temozolomide: a children's oncology group phase I consortium study. J Clin Oncol 2008;26:4921-4927.

26 Ganti R, Skapek S, Zhang J, et al. Expression and genomic status of EGFR and ErbB-2 in alveolar and embryonal rhabdomyosarcoma. Modern Pathol 2006;19:1213-1220.

27 Layfield L, Thompson J, Dodge R, et al. Prognostic indicators for neuroblastoma: stage, grade, DNA ploidy, MIB-1-proliferation index, p53, HER2/neu and EGFR-a survival study. J Surg Oncol 1995;59:21-27.

28 Gambini C, Sementa A, Boni L, et al. Expression of $\mathrm{HER} 2 / \mathrm{neu}$ is uncommon in human neuroblastic tumors and is unrelated to tumor progression. Cancer Immunol Immunother 2003;52:116-120.

29 Ho R, Minturn J, Hishiki T, et al. Proliferation of human neuroblastomas mediated by the epidermal growth factor receptor. Cancer Res 2005;65:9868-9875.

30 Liu S, Tian Y, Chlenski A, et al. Cross-talk between Schwann cells and neuroblasts influences the biology of neuroblastoma xenografts. Am J Pathol 2005;166:891-900.

31 Krams M, Heidebrecht HJ, Hero B, et al. Repp86 expression and outcome in patients with neuroblastoma. J Clin Oncol 2003;21:1810-1818. 